\title{
Quantitative analysis: Specific normal database for the best practice
}

\author{
Gabriel Blacher Grossman, Md, PhD, FASNC,, and Ilo S. Baptista, BSc (Hons.) \\ Physics ${ }^{\mathrm{a}, \mathrm{b}}$ \\ a Nuclear Medicine Department, Hospital Moinhos de Vento, Porto Alegre, Brazil \\ b Cardionuclear, Instituto de Cardiologia, Porto Alegre, Brazil
}

Received Aug 11, 2019; accepted Aug 12, 2019

doi: 10.1007/s12350-019-01902-w

See related article, pp. 1284-1293

The use of dedicated cardiac multi-pinhole cadmium-zinc-telluride (CZT) single-photon emission computerized tomography (SPECT) systems enabled the acquisition of images with a higher number of counts, higher quality images, ultrafast acquisitions, dose reduction, and similar diagnostic accuracy using the same radiotracers that are used for parallel hole SPECT myocardial perfusion imaging (MPI) cameras. Also, CZT systems allows dynamic acquisition of the tomographic images suitable for in vivo assessment of radiotracer kinetics and opens up a new era for myocardial flow and flow reserve measurement using SPECT imaging. ${ }^{1}$ Despite the high cost of these new cameras, its use is increasing around the world.

However, sensitivity of a detection system that uses pinhole collimators is significantly degraded by increasing the distance between the target organ and the detector. $^{2}$ Also, detection geometry of the detectors, depth of the organ, and attenuation artifacts are different reasons for the loss of sensitivity. Current attenuation correction systems help to correct these artifacts by utilizing the X-ray transmission images, which estimate the linear attenuation coefficients of voxels that form the attenuation map. Investigators have reported improvement in the specificity and normalcy rate of attenuationcorrected (AC) images in a general population, without reducing sensitivity. ${ }^{3} \mathrm{CZT}$ cameras are able to detect a

Reprint requests: Gabriel Blacher Grossman, Md, PhD, FASNC, Nuclear Medicine Department, Hospital Moinhos de Vento, Porto

Alegre, Brazil; ggrossman@terra.com.br

J Nucl Cardiol 2021;28:1294-7.

1071-3581/\$34.00

Copyright (C) 2019 American Society of Nuclear Cardiology. larger number of photons per unit of time, but consequently the amount of noise in the acquired image increases proportionally, especially in attenuation corrected images. ${ }^{1}$

Besides the loss of photon sensitivity that decreases with increasing distance from the pinhole collimators, there is a greater variability over the field of view (FOV) than in a parallel-hole camera. In this issue of the Journal, Cuddy-Walsh et al. evaluated if this variation of sensitivity and the spatial gradients will lead to gradients in image noise within the FOV and if there is impact in the efficacy of visual and quantitative image interpretation with a normal database. ${ }^{4}$ Also, it was analyzed if the heterogeneous attenuation of different regions of the heart increases the noise gradient in multi-pinhole camera images and introduces a noise gradient in parallel-hole camera images. These effects could impact the clinical sensitivity of disease detection for different areas of the heart. Therefore, the authors quantified the extent of the noise gradient and evaluated differences between multi-pinhole and standard parallel-hole SPECT cameras regarding image noise gradients and variability within normal databases both with and without attenuation correction. The authors demonstrated that image noise and normal database uncertainty increased from the apex to the base of the heart in attenuation-corrected multi-pinhole SPECT images, but not in non-attenuation corrected images or those acquired with a parallel-hole camera.

This study is interesting and has the virtue to be the first work to evaluate the non-uniform photon sensitivity and the heterogeneous attenuation in cameras on the spatial distribution of image noise. However, when normal database analysis is used, the uncertainty should impact the statistics calculated as part of the normal database development. Thus it should be one of the factors the analysis software uses in evaluating normal/ abnormal images. 

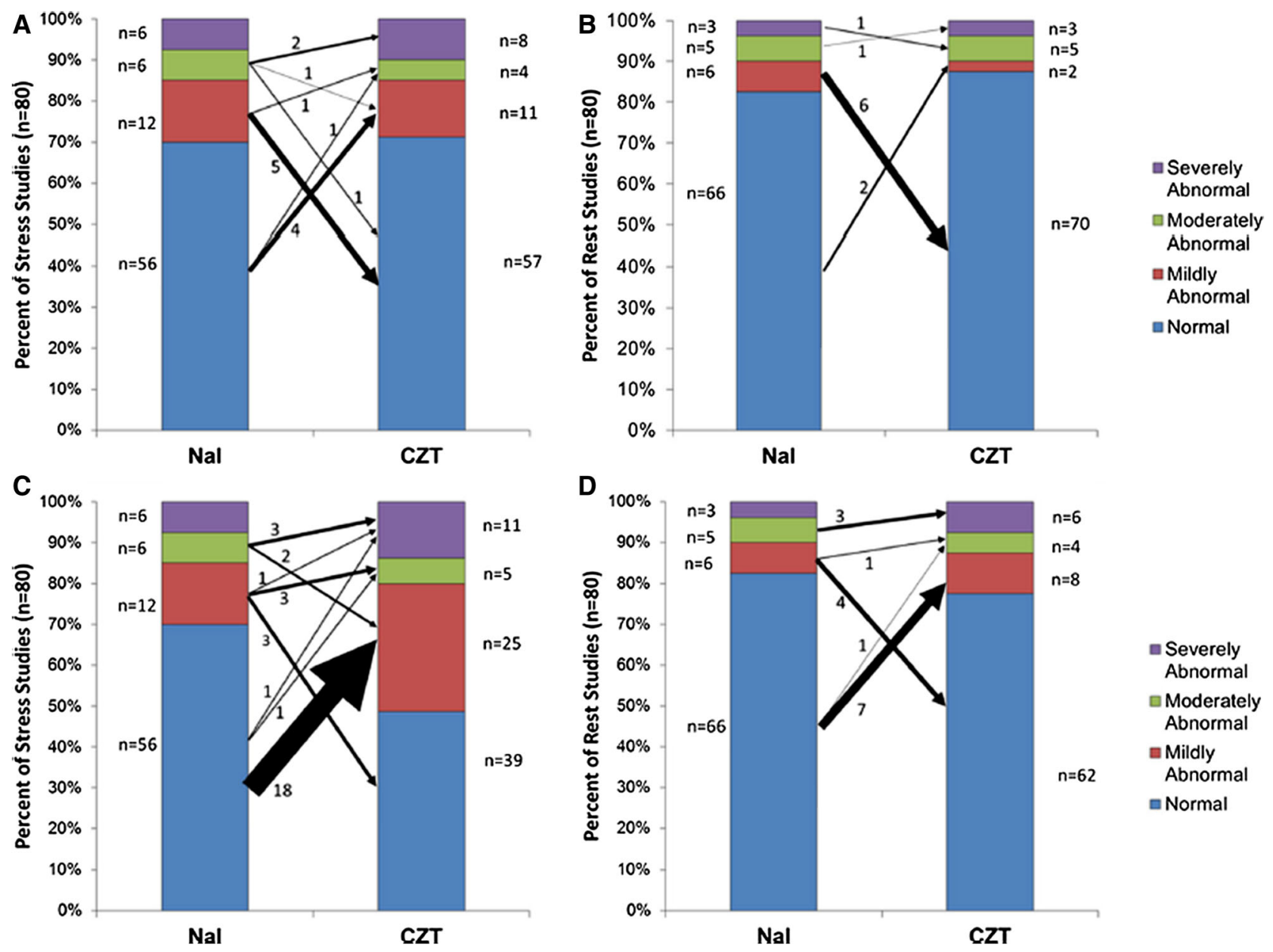

Figure 1. Changes in summed stress (SSS) and rest score (SRS) categories between NaI and CZT showing greater agreement in SSS (A) and SRS (B) if the CZT is referred to the custom database, and greater discordance in SSS (C) and SRS (D) if the CZT is referred to the default database. This figure is reprinted from Kennedy et al. ${ }^{8}$ with permission.

Even though the authors demonstrated that there is an image noise gradient in attenuation-corrected images acquired with multi-pinhole cameras, the clinical impact of this information comparing the extent of ischemia of these studies with non-attenuation-corrected images acquired with multi-pinhole cameras was not analyzed. This investigation evaluated the noise characteristics of the reconstructed images using images that were evaluated normal. No investigation was made into lesion detectability. For this purpose, stress images and different protocols should have been used.

Multiple studies have assessed the diagnostic performance of CZT SPECT with computed tomography (CT) AC, using invasive coronary angiography as a comparator. These studies demonstrated improved diagnostic confidence and accuracy with $\mathrm{CT}$ AC. ${ }^{5-7}$
Recently, Kennedy et al. demonstrated that specifically designed databases for solid-state CZT cardiac SPECT provide accurate quantitation of perfusion scores concordant with those previously validated for conventional $\mathrm{SPECT}^{8}$ (Figures 1 and 2). It is important to point out that the same concordance was not achieved using solely the normal default database available for the conventional system. AC and non-AC CZT scores differed significantly, especially in men. Therefore, even though Cuddy-Walsh et al. demonstrated that noise heterogeneity in multi-pinhole camera images increases with the use of attenuation correction, there is no data showing that this gradient impacts clinical diagnostic accuracy.

Grossman et al. developed a gender-independent stress normal database, and criteria for abnormality for 

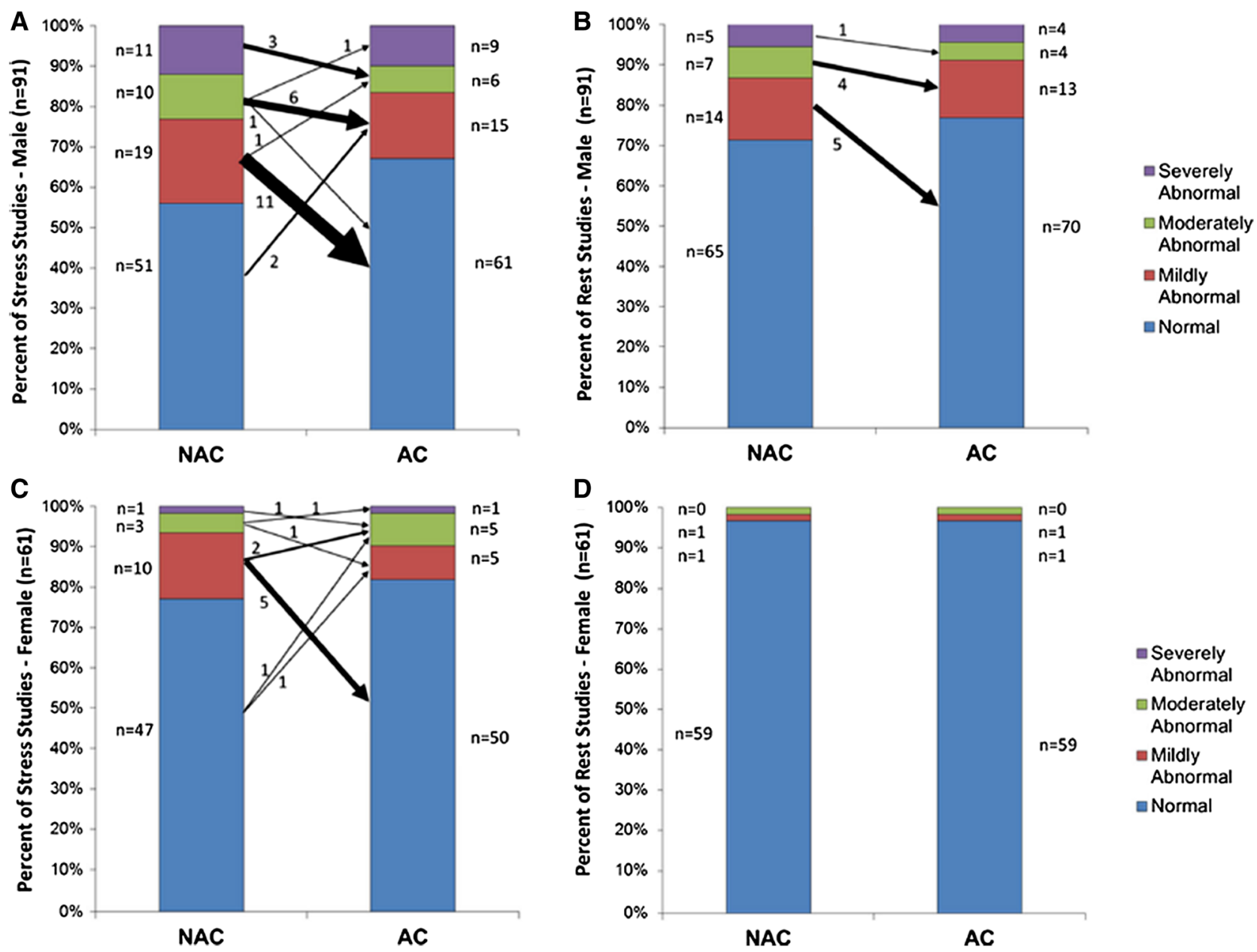

Figure 2. Changes in summed stress (SSS) and rest score (SRS) categories between NAC and AC for the CZT referenced to the custom database. A SSS males. B SRS males. C SSS females. D SRS females. This figure is reprinted from Kennedy et al. ${ }^{8}$ with permission.

attenuation-corrected rest-stress technetium 99m sestamibi same-day myocardial perfusion imaging were developed by evaluation of 112 patients, validated against an obese population of 95 patients from four different clinical sites, and compared with conventional gender-matched database quantification of non-attenuation-corrected studies. ${ }^{9}$ It was demonstrated that attenuation-corrected studies can be quantified with a single gender-independent normal database and a single criterion for abnormality without loss of sensitivity and with significantly better specificity and normalcy rate (Figure 3). However Cuddy-Walsh et al. demonstrated in their study that there are differences in the underlying noise and it is large enough in multi-pinhole SPECT images such that the uncertainty in normal databases is not made independent of gender by attenuation correction. They suggest the use of specific gender databases for attenuation corrected multi-pinhole SPECT studies.

The use of multi-pinhole cameras is growing in nuclear cardiology labs and it is possible to incorporate $\mathrm{AC}$ in these systems. AC enhances clinical interpretation and improves confidence. It also improves quantitative analysis, yielding a significantly higher normalcy rate and specificity without a significant loss in sensitivity. Quantitative analysis is an important tool in nuclear cardiology and ischemia quantification is used for clinical management. When attenuation correction is used, it is necessary to integrate attenuation correction normal databases for quantification. What is really important is that physicians should know that the use of cameraspecific and reconstruction algorithm-specific normal databases for quantitative analysis is the best practice. 


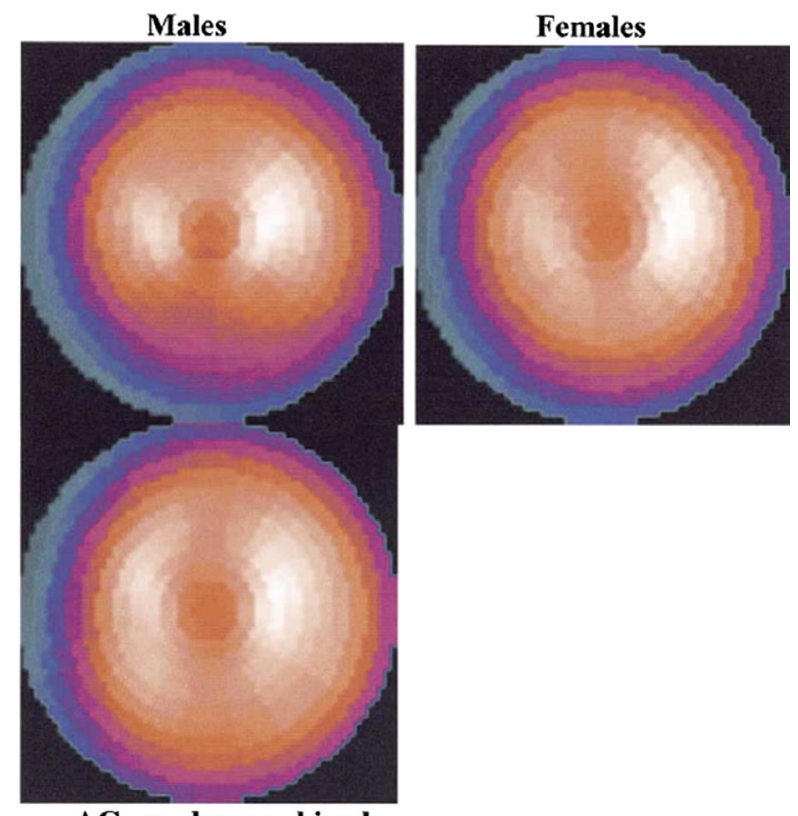

AC, gender-combined

Figure 3. Comparison of gender-matched uncorrected and gender-independent AC mean normal myocardial perfusion distributions. Top, Uncorrected stress polar maps for mean normal 1-day sestamibi: male (left) and female (right) distributions. Bottom, AC gender-combined stress polar map for mean normal myocardial perfusion distribution. Note the increased global uniformity of the AC gender-combined stress polar map. This figure is reprinted from Grossman et al. ${ }^{9}$ with permission.

\section{Disclosure}

Gabriel Blacher Grossman and Ilo S. Baptista have no conflict of interest.

\section{References}

1. Agostini D, Marie P, Ben-Haim S, et al. Performance of cardiac cadmium-zinc-telluride gamma camera imaging in coronary artery disease A review from the cardiovascular committee of the european association of nuclear medicine (EANM). Eur $\mathrm{J}$ Nucl Med Mol Imaging 2016;43:2423-32.

2. Kennedy JA, Israel O, Frenkel A. 3D iteratively reconstructed spatial resolution map and sensitivity characterization of a dedicated cardiac SPECT camera. J Nucl Cardiol 2014;21:443-52.

3. Hendel RC, Corbett JR, Cullom JC, et al. The value and practice of attenuation correction for myocardial perfusion SPECT imaging: A joint position statement from the American Society of Nuclear Cardiology and the Society of Nuclear Medicine. J Nucl Cardiol 2002;43:273-80.

4. Cuddy-Walsh S, Wells R. Noise heterogeneity in attenuationcorrected cardiac SPECT image increases perfusion value uncertainty near the base of the heart. J Nucl Cardiol 2019. https://doi. org/10.1007/s12350-019-01821-w.

5. Esteves F, Galt J, Folks R, Verdes L, Garcia E. Diagnostic performance of low-dose rest/stress tc-99m tetrofosmin myocardial perfusion SPECT using the 530c CZT camera: Quantitative vs visual analysis. J Nucl Cardiol 2014;21:158-65.

6. Caobelli F, Akin M, Thackeray JT, et al. Diagnostic accuracy of cadmium-zinc-telluride-based myocardial perfusion SPECT: Impacto $f$ attenuation correction using a co-registered external computed yomography. Eur Heart $\mathrm{J}$ Cardiovasc Imaging 2016;17:1036-43.

7. van Dijk JD, Mouden M, Ottervanger JP, et al. Value of attenuation correction in stress only myocardial perfusion imaging using CZTSPECT. J Nucl Cardiol 2017;24:395-401.

8. Kennedy JA, Brodov Y, Weinstein AL, et al. The effect of CTbased attenuation correction on the automatic perfusion score of myocardial perfusion imaging using a dedicated cardiac solid-state CZT SPECT/CT. J Nucl Cardiol 2019;26:236-45.

9. Grossman GB, Garcia EV, Bateman TM, et al. Quantitative Tc$99 \mathrm{~m}$ sestamibi attenuation-corrected SPECT: Development and multicenter trial validation of myocardial perfusion stress genderindependent normal database in an obese population. J Nucl Cardiol 2004;11:263-72.

Publisher's Note Springer Nature remains neutral with regard to jurisdictional claims in published maps and institutional affiliations. 